\title{
A small natural molecule promotes mitochondrial fusion through inhibition of the deubiquitinase USP30
}

Wen Yue ${ }^{1,2, *}$, Ziheng Chen ${ }^{1,2, *}$, Haiyang $\mathrm{Liu}^{3}$, Chen $\mathrm{Yan}^{3}$, Ming Chen ${ }^{1,2}$, Du Feng ${ }^{4}$, Chaojun Yan ${ }^{6}$, Hao Wu ${ }^{1,2}$, Lei $\mathrm{Du}^{1,2}$, Yueying Wang ${ }^{1,2}$, Jinhua Liu ${ }^{4}$, Xiaohu Huang ${ }^{5}$, Laixin Xia ${ }^{1}$, Lei Liu ${ }^{1}$, Xiaohui Wang ${ }^{1}$, Haijing Jin ${ }^{1}$, Jun Wang ${ }^{1}$, Zhiyin Song ${ }^{6}$, Xiaojiang $\mathrm{Hao}^{3}$, Quan Chen ${ }^{1,2,4}$

${ }^{I}$ Laboratory of Apoptosis and Mitochondrial Biology, The State Key Laboratory of Biomembrane and Membrane Biotechnology, Institute of Zoology, Chinese Academy of Sciences, Beijing 100101, China; ${ }^{2}$ University of Chinese Academy of Sciences, Beijing 100049, China; ${ }^{3}$ The State Key Laboratory of Phytochemistry and Plant Resources in West China, Kunming Institute of Botany, Chinese Academy of Sciences, Kunming, Yunnan 650204, China; ${ }^{4}$ College of Life Sciences, Nankai University, Tianjin 300071, China; ${ }^{5}$ State Key Laboratory of Biomembrane and Membrane Biotechnology, Institute of Molecular Medicine, Peking-Tsinghua Center for Life Sciences, Peking University, Beijing 100871, China; ${ }^{6}$ College of Life Sciences, Wuhan University, Wuhan, Hubei 430072, China

Mitochondrial fusion is a highly coordinated process that mixes and unifies the mitochondrial compartment for normal mitochondrial functions and mitochondrial DNA inheritance. Dysregulated mitochondrial fusion causes mitochondrial fragmentation, abnormal mitochondrial physiology and inheritance, and has been causally linked with a number of neuronal diseases. Here, we identified a diterpenoid derivative 15-oxospiramilactone (S3) that potently induced mitochondrial fusion to restore the mitochondrial network and oxidative respiration in cells that are deficient in either Mfn1 or Mfn2. A mitochondria-localized deubiquitinase USP30 is a target of S3. The inhibition of USP30 by S3 leads to an increase of non-degradative ubiquitination of Mfn1/2, which enhances Mfn1 and Mfn2 activity and promotes mitochondrial fusion. Thus, through the use of an inhibitor of USP30, our study uncovers an unconventional function of non-degradative ubiquitination of Mfns in promoting mitochondrial fusion.

Keywords: mitochondrial fusion; mitofusin 1/2; deubiquitinase USP30; 15-Oxospiramilactone

Cell Research (2014) 24:482-496. doi:10.1038/cr.2014.20; published online 11 February 2014

\section{Introduction}

Mitochondria are highly dynamic organelles undergoing constant fission and fusion to maintain normal morphology, which is important for mitochondrial functions and fundamental cellular processes [1,2]. Several key molecules are known to mediate this complex process. Mfn1 and Mfn2 are GTPases anchored to the outer mitochondrial membrane [3]. The homo- and heterodimer-

\footnotetext{
*These two authors contributed equally to this work.

Correspondence: Quan Chen ${ }^{\mathrm{a}}$, Xiaojiang $\mathrm{Hao}^{\mathrm{b}}$

${ }^{\mathrm{a}}$ Tel: +86-010-64807322

E-mail: chenq@ioz.ac.cn

${ }^{\mathrm{b}} \mathrm{Tel}$ : +86-0871-5219684

E-mail: haoxj@mail.kib.ac.cn

Received 30 March 2013; revised 25 October 2013; accepted 26 November 2013; published online 11 February 2014
}

ization between Mfn1 and Mfn2 are essential for the tethering of adjacent mitochondria before the complete fusion of neighboring mitochondria [4-7]. Another GTPase, OPA1, localized in the intermembrane space and the inner membrane of mitochondria, is responsible for fusion of the inner mitochondrial membrane $[8,9]$. Dysregulated activity of fusion mediators leads to extensive mitochondrial fragmentation and severe mitochondrial dysfunction, such as loss of mitochondrial potential and defect in respiratory capacity, possibly as a consequence of the loss of mitochondrial DNA (mtDNA) in individual mitochondria $[10,11]$. Mice lacking Mfn1, Mfn2 or OPA1 die at an early embryonic stage, indicating that fusion factors are strictly required during early development $[12,13]$. Moreover, point mutations in Mfn 2 or OPA1 lead to severe neurodegenerative diseases such as Charcot-Marie-Tooth type 2A and dominant optic atrophy $[14,15]$. On the other hand, mitochondrial fission is 
regulated by another GTPase family member, dynaminrelated protein 1 (Drp1) [16]. Drp1 is recruited to mitochondria by mitochondrial fission factor or MiD49/51 and self-assembles into spirals surrounding the mitochondria to drive membrane constriction and fission [16-19]. Cells lacking Drp1 showed deficiency in mitochondrial fission and exhibited abnormally elongated mitochondria [20]. Despite the importance of mitochondrial dynamics in many biological processes, including embryo development, neuron degeneration, cellular metabolism and cell survival, the regulatory mechanisms by which the mitochondrial fusion and fission processes are orchestrated to fulfill these complex functions remain poorly understood. In particular, little is known about how mitochondrial fusion is regulated. Recently, the mitofusin binding protein (MIB) was found to be a negative regulator of Mfn1 [21].

Early studies in yeast revealed that ubiquitination affects mitochondrial morphology and mitochondrial inheritance [22]. In mammalian cells, several E3 ligases were found to localize at or translocate to mitochondria to mediate ubiquitination of Mfn1/2 or Drp1 for their degradation [23-30]. Recent research has shown that ubiquitination not only leads the protein substrate to the proteasome or lysosome for degradation, but also regulates various cellular functions, including signal transduction, endocytic trafficking and DNA repair without affecting protein stability [31]. Many proteins contain ubiquitinbinding domains or motifs that function as ubiquitin receptors for protein-protein interactions [32]. It is thus intriguing to determine whether such a mechanism is involved in regulating mitochondrial dynamics. Protein ubiquitination is a reversible process and this reversibility is accomplished by deubiquitinases that remove ubiquitin from their substrates [33]. There are about 100 deubiquitinases in the human genome, and more than half of human deubiquitinases belong to the ubiquitinspecific protease (USP) subfamily that contains critical cysteine and histidine residues in the reactive center [34]. A number of deubiquitinases are reported to play critical roles in diverse cellular and physiological functions such as cell signaling, histone modification and so on $[35,36]$. Abnormal deubiquitinase activity is closely related to tumor cell survival as these enzymes modulate TGF- $\beta$, Wnt and TNF signaling pathways [37]. Several small molecules that inhibit distinct deubiquitinases have been identified, and these small molecules would be useful tools for studying the molecular mechanisms underlying the actions of these deubiquitinases, in addition to their potential therapeutic applications [38, 39].

In the present study, we identified a small natural derivative $\mathrm{S} 3$, which potently activated mitochondrial fusion accompanied by restoration of normal mitochondrial function. We found that S3-induced inhibition of USP30, a mitochondria-localized deubiquitinase, increased the ubiquitination of Mfn1 and Mfn2 without affecting their protein levels. This non-degradative ubiquitination of Mfns is involved in regulation of mitochondrial fusion.

\section{Results}

S3-induced mitochondrial re-networking in the absence of either Mfn 1 or Mfn2

To understand the regulatory mechanism of mitochondrial fusion and fission, we screened for small molecules that could induce the elongation of mitochondria in Mfn1-knockout $\left(\mathrm{Mfn}^{-/-}\right) \mathrm{MEF}$ cells. In these cells, small-fragmented mitochondria dispersed within the cell and the elongation of mitochondria was readily detectable. From the 300 compounds obtained, we identified 15-oxospiramilactone, a diterpenoid derivative (named S3 hereafter) [40], which could induce remarkable mitochondrial elongation in cells that lack Mfn1. Using a mitochondrial matrix-targeted DsRed protein, we monitored mitochondrial morphological changes at the singlecell level in real time (Figure 1A). Following the addition of $5 \mu \mathrm{M} \mathrm{S} 3$, the mitochondrial morphology changed from spheres to highly-interconnected filaments, and the disrupted mitochondrial network was rebuilt within $2 \mathrm{~h}$. S3-induced mitochondrial morphological change occurs in a dose- and time-dependent manner (Figure 1A and 1B). Treatment with $2 \mu \mathrm{M} \mathrm{S} 3$ for $24 \mathrm{~h}$ could efficiently induce mitochondrial elongation in approximately $80 \%$ of the cells without affecting the cell viability, while concentration higher than $5 \mu \mathrm{M}$ could kill the cells through apoptosis [38] (Supplementary information, Figure S1). We thus chose this dose and timepoint for further experiments.

To demonstrate that $\mathrm{S} 3$ induces complete mitochondrial fusion, but not mere mitochondria association or aggregation, we carried out an ultrastructural analysis by electron microscopy (EM). EM pictures showed that following S3 treatment, the dot-like or rod-like mitochondria in $\mathrm{Mfn}^{-/-}$or $\mathrm{Mfn} 2^{-/-} \mathrm{MEF}$ cells were changed to an interconnected mitochondrial network with branching (Figure 1C), indicating that S3 induces complete and coordinated mitochondrial fusion.

We next sought to understand if S3-induced mitochondrial fusion is dependent on the core mitochondrial fusion machinery. We compared the effects of S3 treatment on mitochondrial morphology in $\mathrm{WT}, \mathrm{Mfn}^{-1-}$, $\mathrm{Mfn}^{-/-}$, Mfn1/2-double knockout (Mfn Dko) and OPA1knockout $\left(\mathrm{OPA}^{-/}\right)$MEF cells. All mitochondria in these mutant cells were fragmented and dot-like or rod-like as described earlier. We found that S3 induced a remarkable 
A

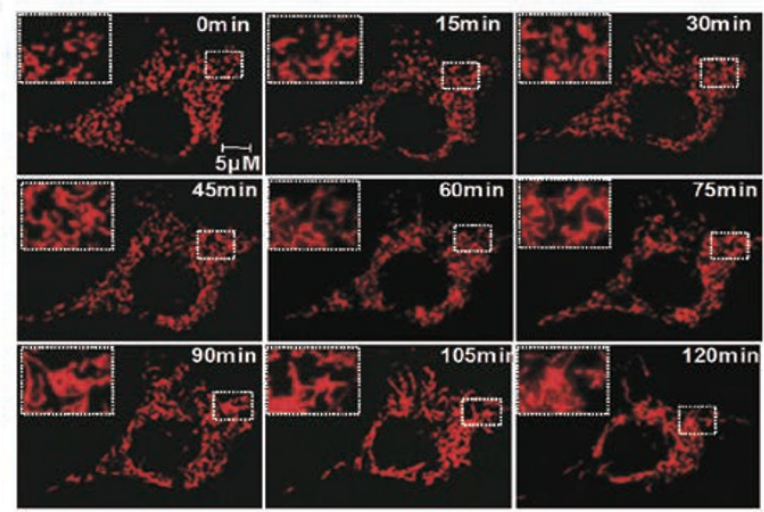

B

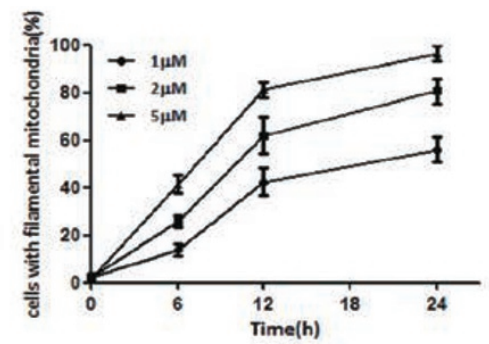

C
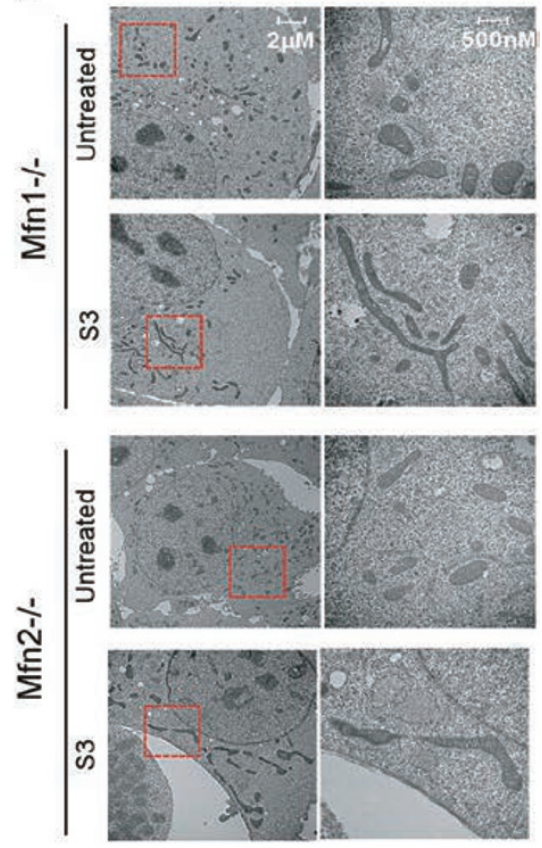

D

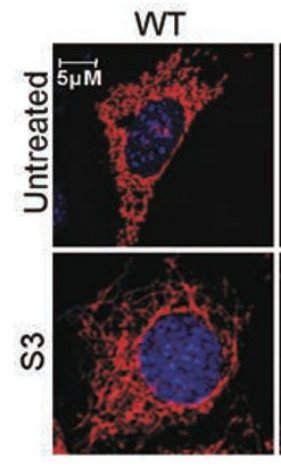

Mfn1 -/-

\section{Mfn2 -/-}
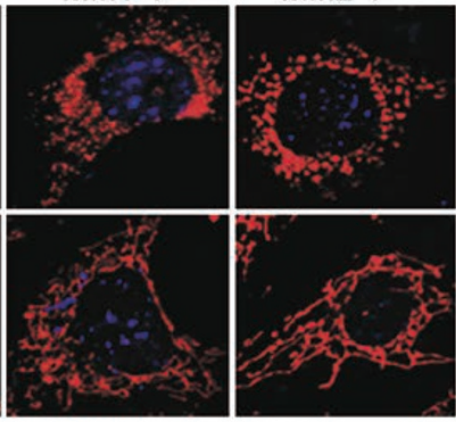

Mfn Dko OPA1 -/-
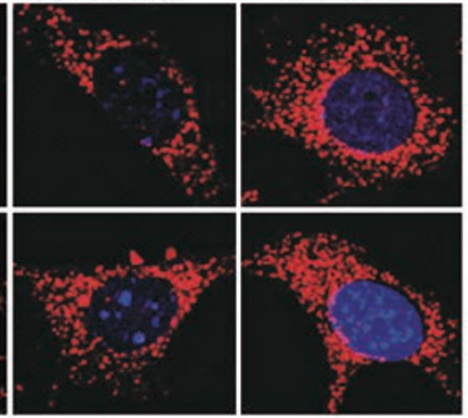

E

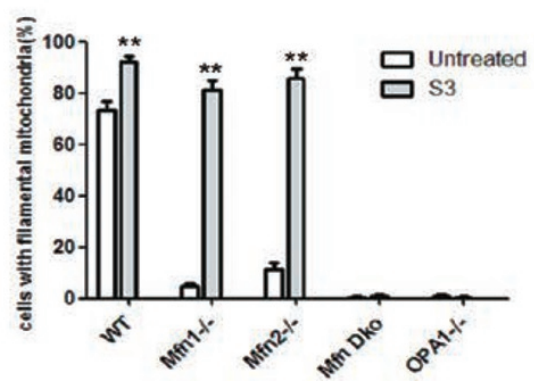

Figure $1 \mathrm{~S} 3$ induces mitochondrial elongation. (A) An Mfn $1^{-1-}$ MEF cell transfected with MitoDsRed and treated with $5 \mu \mathrm{M}$ S3 was monitored by confocal microscopy for $2 \mathrm{~h}$ with an interval of $15 \mathrm{~min}$. The scale bar $(5 \mu \mathrm{M})$ is shown on the top left. (B) Quantification of cells with connected and tubular mitochondria at the indicated time points after exposure to different concentrations of S3. Data represent the mean \pm SD of three independent experiments, each with > 200 cells counted per condition. (C) Ultrastructural analysis of mitochondria of $\mathrm{Mfn}^{-/-}$and Mfn2 $2^{-/-} \mathrm{MEF}$ cells. Cells were untreated or treated with $2 \mu \mathrm{M} \mathrm{S} 3$ for $24 \mathrm{~h}$ and fixed for electron microscopy analysis. (D) Mitochondrial morphologies of WT, Mfn $1^{-{ }^{-}}$, Mfn2 $2^{-/-}$, Mfn Dko and OPA ${ }^{1^{-1}}$ MEF cells untreated or treated with $2 \mu \mathrm{M} \mathrm{S} 3$ for $24 \mathrm{~h}$. Cells were transfected with MitoDsRed for visualization of mitochondria. (E) Quantification of the indicated cells with connected and tubular mitochondria before and after $2 \mu \mathrm{M}$ S3 treatment for $24 \mathrm{~h}$. Data represent mean \pm SD of three independent experiments, each with $>200$ cells counted per condition. ${ }^{* *} P<0.001$, unpaired $t$ test, two tailed. 
mitochondrial elongation in cells that were deficient in either Mfn1 or Mfn2 (Figure 1D and 1E). Actually, in most of these S3-treated cells, mitochondria were well interconnected to form a mitochondrial reticulum similar to that observed in WT cells. However, S3 failed to change the mitochondrial morphology when both Mfn1 and Mfn2 were deleted, or in cells that were deficient in OPA1. These data demonstrate that OPA1 and at least one of the Mfns are required for S3-induced mitochondrial fusion.

To further clarify whether S3-induced mitochondrial fusion requires functional Mfns, we overexpressed GTPase mutants of Mfn1 and Mfn2 in Mfn Dko cells. As shown in Supplementary information, Figure S2, when WT Mfn1 or Mfn2 was expressed in Mfn Dko cells, mitochondria became highly fused and aggregated. After S3 treatment, the mitochondrial morphology did not show much change due to the already very condensed structure. However, when GTPase mutants of Mfns (Mfn1 K88T or Mfn2 K109A) were expressed in Mfn Dko cells, mitochondria remained fragmented in the absence or presence of S3, which indicates that S3-induced mitochondrial fusion is dependent on the normal function of Mfns. These data showed that S3 requires functional Mfns (either Mfn1 or Mfn2) to induce mitochondrial fusion.

Restoration of mitochondrial oxidative phosphorylation and the distribution of mtDNA

Previous studies have revealed that cells that are deficient in Mfn1, Mfn2 or OPA1 have impaired mitochondrial functions [10, 11]. It is therefore important to determine whether $\mathrm{S} 3$ can restore mitochondrial functions in these cells. We first monitored $\Delta \psi_{\mathrm{m}}$ using a mitochondriaspecific dye MitoTracker Red, whose sequestration into the mitochondria is dependent on $\Delta \psi_{\mathrm{m}}$. Cells were transfected with mitoGFP, which is located in the mitochondrial matrix independent of $\Delta \psi_{\mathrm{m}}$, and then stained with MitoTracker Red. In WT cells, GFP-marked mitochondria were uniformly stained by MitoTracker Red and the two fluorophores were completely colocalized (Figure 2A). However, $\mathrm{Mfn}^{-/-}$, Mfn2 ${ }^{-/-}$, Mfn Dko and OPA1 $1^{-/}$ cells showed heterogeneity in $\Delta \psi_{\mathrm{m}}$. A portion of the mitochondria in these cells were marked only by mitoGFP, but not by MitoTracker Red. Importantly, following S3 treatment, the mitochondria fused and the mitochondrial network remerged with the overall $\Delta \psi_{\mathrm{m}}$ recovered in $\mathrm{Mfn}^{-/-}$and $\mathrm{Mfn}^{-/-}$cells (Figure $2 \mathrm{~A}$ ), indicating that the $\Delta \psi_{\mathrm{m}}$ had been restored by the whole mitochondrial reticulum. However, $\mathrm{S} 3$ failed to restore the loss of $\Delta \psi_{\mathrm{m}}$ in Mfn Dko or OPA $1^{-/-}$cells, which is consistent with their unchanged mitochondrial morphology (Figure 1D and
1E).

We next measured the cellular ATP levels. In Mfn1 $1^{-1}$ or $\mathrm{Mfn} 2^{-/-}$cells, S3 induced a significant increase in the ATP levels (Figure 2B). However, there was no difference between the untreated and treated Mfn Dko cells (Figure 2B), indicating that the increase in ATP levels is related to the restoration of the mitochondrial network. To verify that the increase of ATP level is due to the increase of mitochondrial oxidative phosphorylation (OXPHOS), we estimated the OXPHOS capacity as previously described [41] (Figure 2C). A substrate of OXPHOS, methylpyruvate (MeP), was added to the adherent cells to activate respiration and the ATP level was measured at an interval of $5 \mathrm{~min}$. Oligomycin, an inhibitor of ATP synthase, was used to confirm that the ATP level increase is derived from mitochondria.

Interestingly, untreated $\mathrm{Mfn}^{-/-}, \mathrm{Mfn}^{-/-}$or Mfn Dko cells showed weak or no increase in the ATP level upon $\mathrm{MeP}$ treatment, as previously reported [41]. However, after S3 treatment, $\mathrm{Mfn}^{-/-}$and $\mathrm{Mfn}^{-/-}$cells showed a significant increase in the level of ATP when treated with MeP, while the ATP level of Mfn Dko cells still remained at the basal level. These results suggest that the increase in the cellular ATP level occurred mainly because of the enhanced ATP production from mitochondria.

A distinguishing feature of mitochondria is the existence of mitochondrial DNA (mtDNA), which encode components of the electron transport chain. Previous reports have shown that mitochondrial fusion is important for the maintenance of mtDNA, and that cells that are deficient in Mfn1 or Mfn2 have abnormal mtDNA inheritance $[42,43]$. Using a specific antibody that recognizes DNA, we found that in $\mathrm{Mfn}^{-/-}$or $\mathrm{Mfn}^{-/-}$or $\mathrm{OPA} 1^{-/-}$cells, a portion of the mitochondria lack $\mathrm{mtD}$ NA, which is consistent with previous reports $[42,43]$. As expected, in $\mathrm{S} 3$-treated $\mathrm{Mfn}^{-/-}$or $\mathrm{Mfn}^{-/-}$cells, the mitochondrial network was rebuilt and the mtDNA was distributed throughout the mitochondrial network (Figure $2 \mathrm{D}$ and $2 \mathrm{E}$ ). In contrast, in S3-treated $\mathrm{OPA}^{-/-}$cells, this did not occur due to the complete lack of mitochondrial fusion. We also measured the numbers of mtDNA copies in untreated and S3-treated cells. Our results showed that the overall mtDNA copy numbers were not changed after S3 treatment (Supplementary information, Figure S3). Clearly, S3 only induced redistribution of mtDNA through restoration of the mitochondrial network without affecting the total mtDNA copy number.

\section{S3-induced activation of mitochondrial fusion re- networks mitochondria}

Mitochondrial morphology is regulated by two opposing processes, namely fusion and fission. S3-induced 

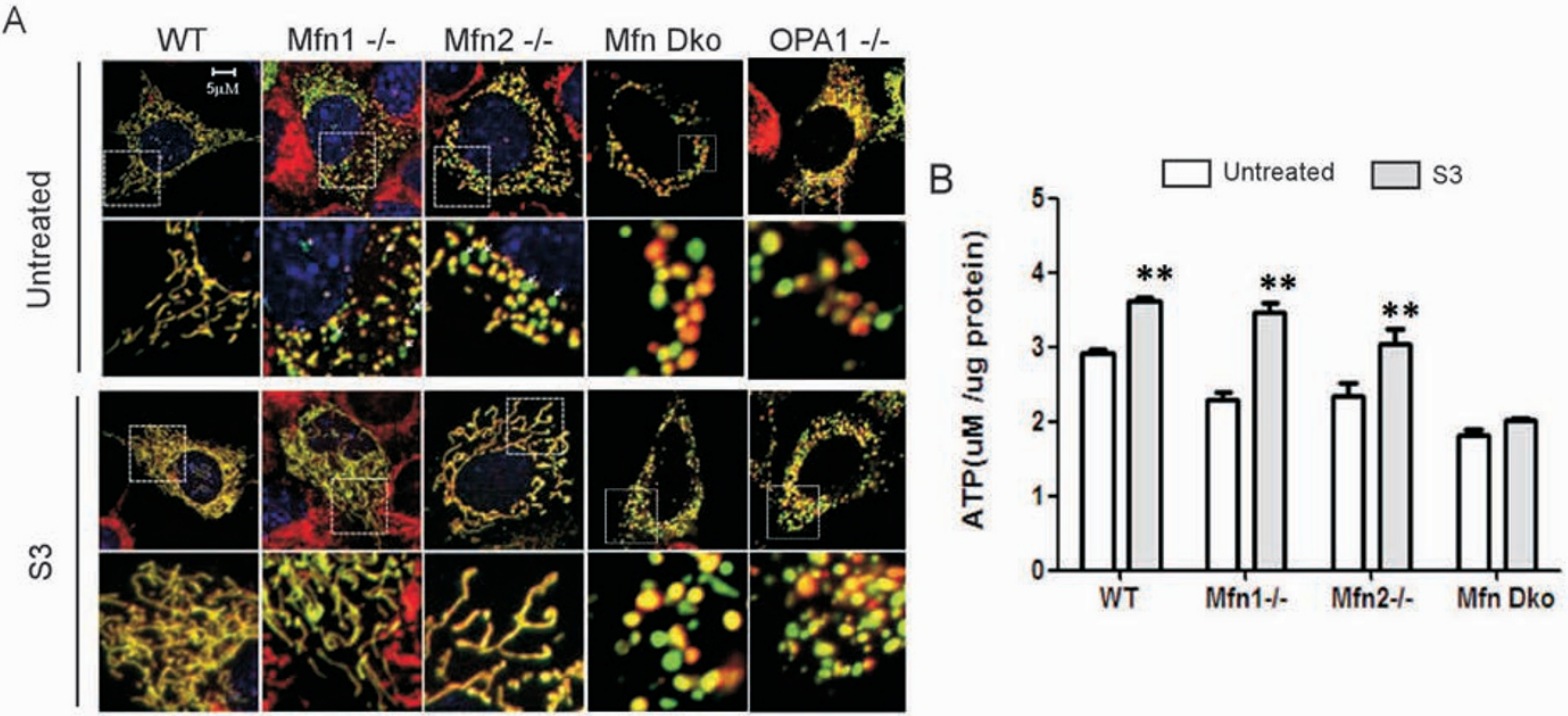

$\mathrm{C}$
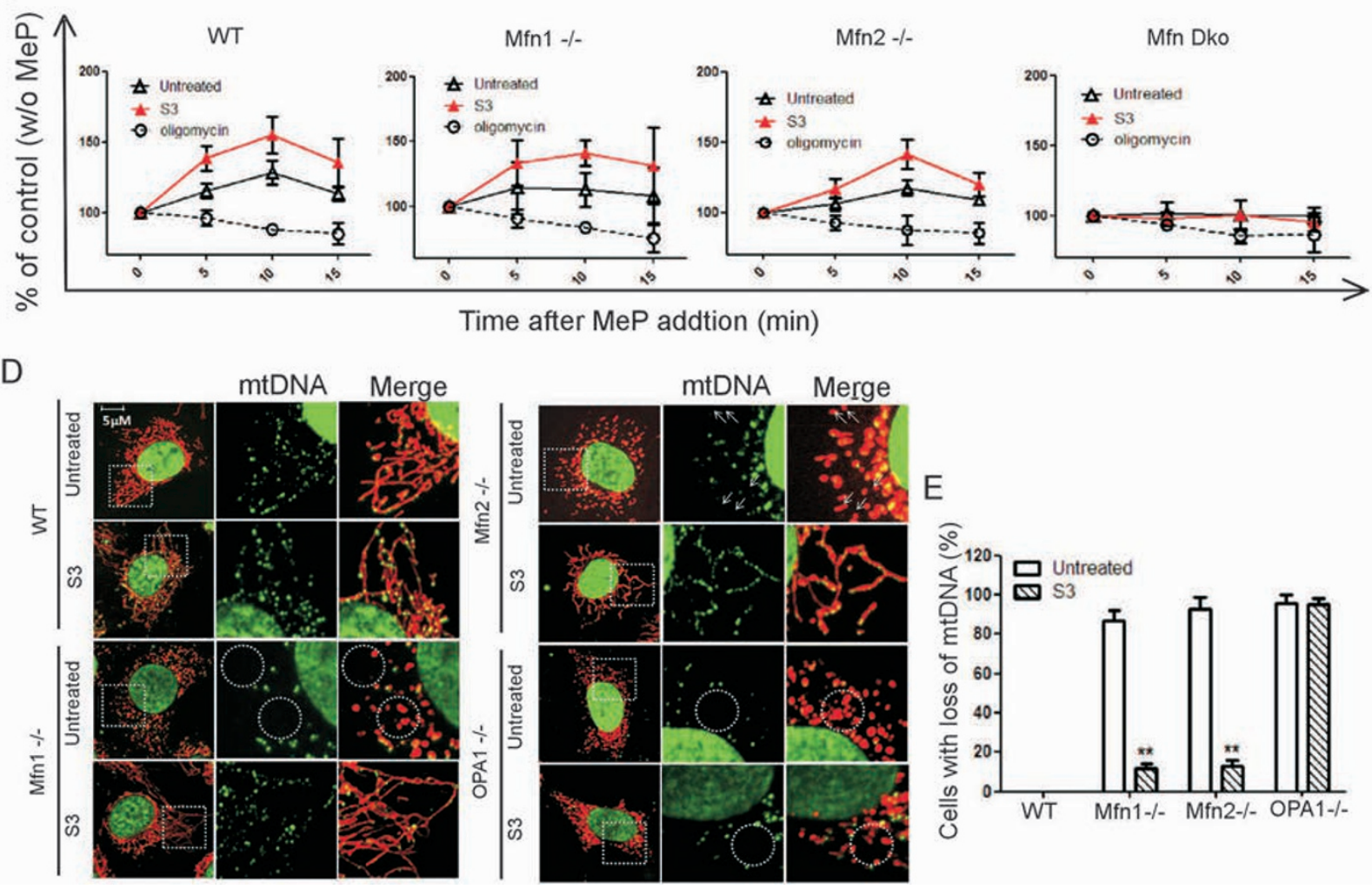

Figure 2 S3 treatment restores mitochondrial functions. (A) MEF cells of the indicated genotypes expressing mitochondriatargeted GFP (green) were untreated or treated with $2 \mu \mathrm{M} \mathrm{S3}$ for $24 \mathrm{~h}$, and then stained with MitoTracker Red (red), whose sequestration into mitochondria is dependent on membrane potential. (B) Total ATP levels in WT, Mfn $1^{-/-}$, Mfn2 $2^{-/-}$and Mfn Dko cells untreated or treated with $2 \mu \mathrm{M} \mathrm{S} 3$ for $24 \mathrm{~h}$. Data represent the mean \pm SD of four independent experiments. ${ }^{* *} P<$ 0.001 , unpaired $t$ test, two tailed. (C) Measurement of mitochondrial OXPHOS capacity in WT, Mfn1 $1^{-/-}$, Mfn2 $2^{-/-}$and Mfn Dko cells. Cells were pretreated with $2 \mu \mathrm{M} \mathrm{S} 3$ for $24 \mathrm{~h}$, and then $10 \mathrm{mM}$ MeP was added into the medium. Total ATP levels were assayed after $5 \mathrm{~min}, 10 \mathrm{~min}$ and $15 \mathrm{~min}$. Also, cells were treated with oligomycin $(10 \mu \mathrm{M})$ and MeP to confirm the detected ATP production is originated from the mitochondria. Data represent the mean \pm SD of five independent experiments. (D) MEFs of the indicated genotypes were untreated or treated with $2 \mu \mathrm{M} \mathrm{S3}$ for $24 \mathrm{~h}$, and were examined for colocalization of mitochondria (MitoDsRed, red) and mtDNA nucleotides (anti-DNA, green). Circles and arrows highlight examples of mitochondria without mtDNA nucleotides. (E) Quantification of cells with loss of mtDNA in WT, Mfn1 ${ }^{-1-}$, Mfn2 ${ }^{-/-}$and OPA1 ${ }^{-1-}$ MEFs. Data represent mean \pm SD of at least three independent experiments. ${ }^{* *} P<0.001$, unpaired $t$ test, two tailed. 
re-networking of mitochondria could be due to either activation of fusion or inhibition of fission. To clarify this question, we first assayed the rate of mitochondrial fusion using a mitochondria-targeted photoactive fluorescence protein, Mito-PAGFP, as previously described [44]. After activation by $405 \mathrm{nM}$ exciting light, activated Mito-PAGFP will diffuse to other mitochondria accompanied by the occurrence of mitochondrial fusion. Thus the rate of fusion can be calculated by the decline in fluorescence intensity. In $\mathrm{Mfn}^{-/-}$or $\mathrm{Mfn}^{-/-}$cells, due to the lack of fusion, the intensity of fluorescence remained stable over $3 \mathrm{~h}$. Strikingly, upon S3 treatment, the rate of mitochondrial fusion dramatically increased to a level that is comparable to that of the WT cells (Figure 3), as reflected by the rapid diffusion of fluorescence within the mitochondrial network. Overexpression of Drp1 did not decelerate the rate of mitochondrial fusion in both S3treated and untreated $\mathrm{Mfn}^{1^{-/}}$cells (Figure 3). In contrast, there is no decrease of fluorescence intensity in Mfn Dko cells when treated with S3 (Figure 3).

To further testify whether S3-induced mitochondrial fusion is due to the inhibition of Drp1, which is responsible for mitochondrial fission, we overexpressed Drp1 in WT, Mfn $1^{--}$and $\mathrm{Mfn}^{-/-}$cells (Supplementary information, Figure S4A and S4B). The results showed that Drp1 could not prevent S3-induced mitochondrial fusion. S3 did not affect either the localization or GTPase activity of Drp1 (Supplementary information, Figure S4C and S4D). As the phosphorylation of Drp1 also regulates its activity in the process of mitochondrial fission, we checked the phosphorylation status of Drp1 after S3 treatment and did not find any change (Supplementary information, Figure S4E). Taken together, we conclude that S3 clearly enhances mitochondrial fusion without affecting the activity of the main fission component Drp1.

\section{Deubiquitinase USP30 is a target of S3 in S3-induced mitochondrial fusion}

To understand the molecular mechanism of S3induced mitochondrial fusion, we checked the protein levels of Mfn1 and Mfn2, which are the master factors in this process. However, we did not detect any significant change in Mfn1 and Mfn2 protein levels even after a prolonged period of S3 treatment (Figure 4A). Interestingly, the ubiquitination of both Mfn1 and Mfn2 increased after S3 treatment (Figure 4B), suggesting that S3 may regulate the function of $\mathrm{Mfn} 1 / 2$ via induction of ubiquitination. The cycloheximide (CHX) chase assay revealed that $\mathrm{S} 3$ has no effect on the degradation of $\mathrm{Mfn} 1 / 2$ (Figure $4 \mathrm{C})$.

It is of interest to note that USP30 is a deubiquitinase localized on mitochondria and can affect mitochondrial morphology in an $\mathrm{Mfn} 1 / 2$-dependent manner [45]. Then we synthesized a Biotin-modified S3 to test whether S3 directly binds to USP30 (Supplementary information, Figure S5). We incubated the cell lysates with Biotin-S3 and found that Myc-tagged USP30 could be pulled down by Biotin-S3, confirming the direct interaction between USP30 and S3 (Figure 4D). As S3 is a "thiol-Michael addition" molecule, it is likely that Biotin-S3 binds to cysteine in the catalytic domain of USP30. Several mutants of USP30 were generated, each containing a point mutation in the catalytic domain of USP30 (Figure 4E). Among them, two mutants (C77S and H452A) involve substitution of residues that are critical for deubiquitinase activity, and the other two (C234S and C284S) involve cysteine residues that were randomly chosen, and thus serve as controls. The binding of S3 to USP30 was abolished when $\mathrm{C} 77$ was mutated, while other mutations of cysteine or histidine residues failed to abolish the binding. Thus, we concluded that $\mathrm{C} 77$ in the catalytic domain of USP30 is the main binding site of Biotin-S3. We also showed that $\mathrm{S} 3$ could inhibit the deubiquitinase activity of USP30 in vitro (Supplementary information, Figure S6), as assayed by its cleavage of Lys48- and Lys63linked Ub4 chains to produce ubiquitin trimers. Taken together, these data show that S3 directly inhibits the deubiquitinase activity of USP30 through its interaction with the cysteine residue in the catalytic domain.

We next examined whether USP30 inhibition is responsible for the mitochondrial fusion induced by $\mathrm{S} 3$ (Figure 5A and 5B). $\mathrm{Mfn}^{-/}$and $\mathrm{Mfn}^{-/-}$cells were transfected with WT USP30 or a USP30 mutant (USP30$\mathrm{C} 77 \mathrm{~S}$ ) and the percentage of cells with filamental mitochondria was analyzed following S3 treatment. In untransfected $\mathrm{Mfn}^{-/-}$or $\mathrm{Mfn} 2^{-/-}$cells, approximately $80 \%$ of the cells contained fused mitochondria after S3 treatment, whereas only $\sim 35 \%$ of $\mathrm{Mfn}^{-/-}$cells and $\sim 38 \%$ of $\mathrm{Mfn}^{-/-}$cells that were transfected with USP30 showed this phenotype. In contrast, overexpression of USP30-C77S did not affect S3-induced mitochondrial fusion in $\mathrm{Mfn}^{-/-}$or $\mathrm{Mfn}^{-/-}$cells, indicating that $\mathrm{S} 3-$ induced mitochondrial fusion was partially inhibited by WT, but not by the inactive form of USP30. These data suggest that S3 may induce mitochondrial fusion via inhibition of USP30 activity.

To confirm that USP30 affects mitochondrial fusion in the absence of Mfn1 or Mfn2, we employed the knockdown approach to specifically downregulate its expression by shRNAs in $\mathrm{Mfn}^{-/-}$or $\mathrm{Mfn} 2^{-/-}$cells (Figure 5C, 5D and Supplementary information, Figure S7A). Interestingly, we found that knockdown of USP30 in $\mathrm{Mfn} 1^{-/-}$and $\mathrm{Mfn} 2^{-/-}$cells resulted in significant promotion of mitochondrial fusion (Figure 5D), which 


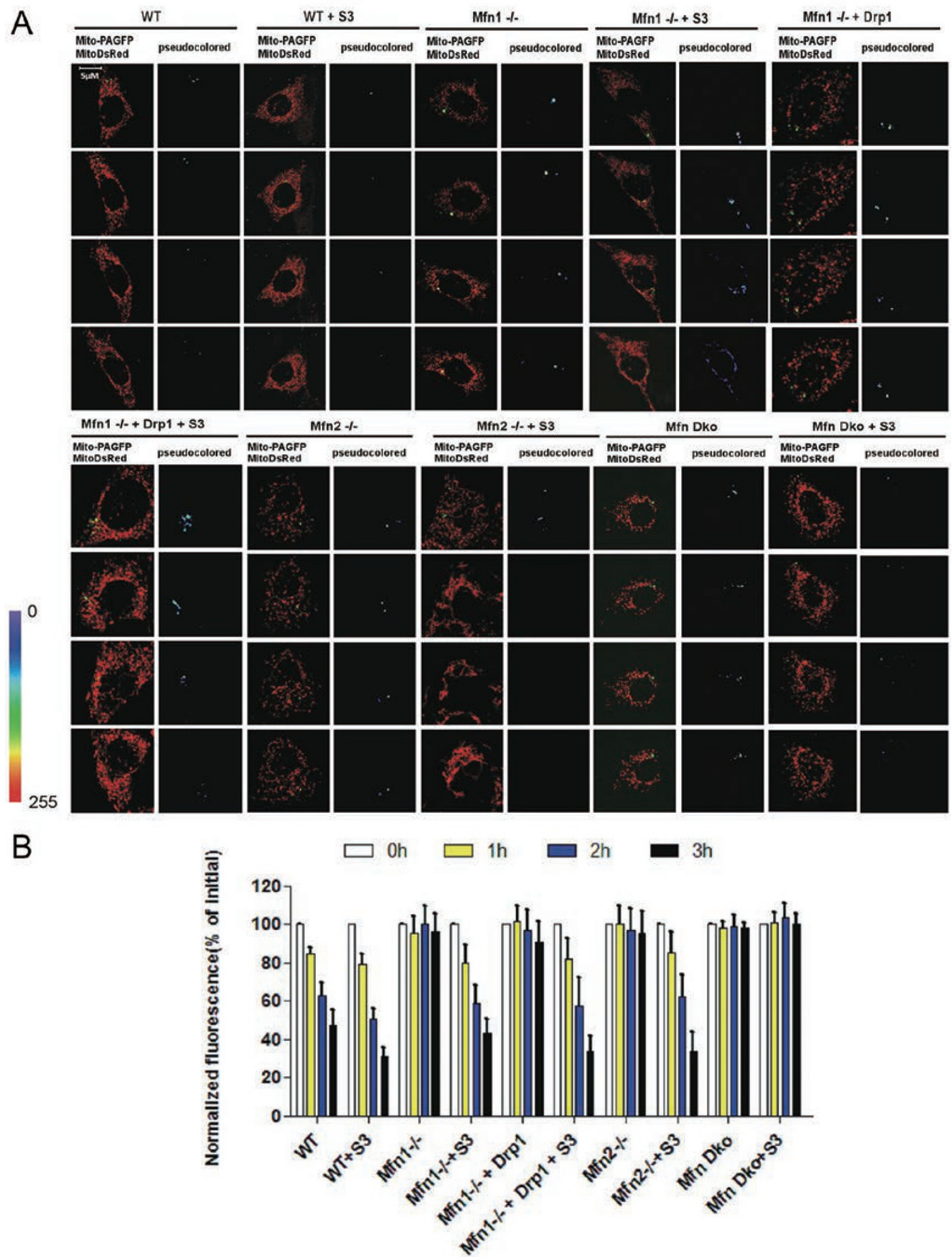

Figure 3 S3 activates mitochondrial fusion. (A) Visualization and (B) quantification of mitochondrial fusion using Mito-PAGFP (green) and MitoDsRed (red) in WT, Mfn $1^{-/-}$, Mfn $1^{-/-}$with Drp1 overexpression, Mfn2 ${ }^{-/}$and Mfn Dko MEFs untreated or treated with $2 \mu \mathrm{M} \mathrm{S} 3$ for $24 \mathrm{~h}$. Cells were co-transfected with Mito-PAGFP and MitoDsRed. Selected regions were photoactivated using $405 \mathrm{~nm}$ light, followed by whole-cell imaging with $488 \mathrm{~nm}$ light. Mitochondrial morphology is shown by MitoDsRed (left panel) and diffusion of Mito-PAGFP is shown in a pseudocolor manner (right panel). Pseudocolor bar indicates arbitrary fluorescence units. Post-acquisition processing and analysis were performed by using ImagePro Plus software. The data represent mean \pm SD of at least three independent experiments, each with $>15$ cells analyzed per condition. Student's $t$-test was conducted between untreated and S3-treated groups in WT, Mfn1 $1^{-1-}, \mathrm{Mfn}^{-1-}$ with Drp1 overexpression, Mfn2 ${ }^{-/-}$and Mfn Dko cells. $P<0.05$, unpaired, two-tailed. 
A

B
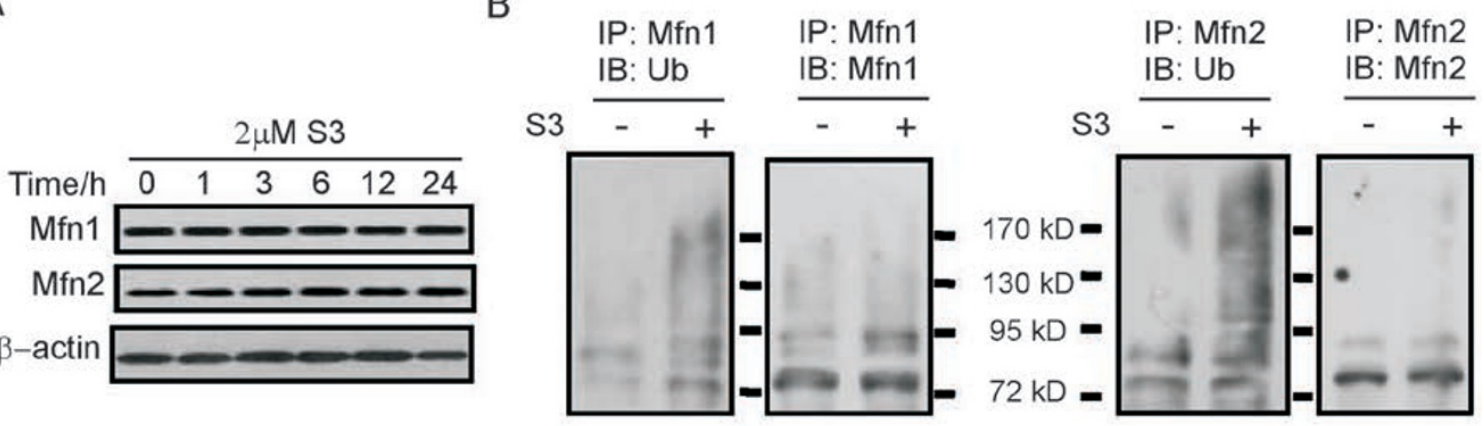

C
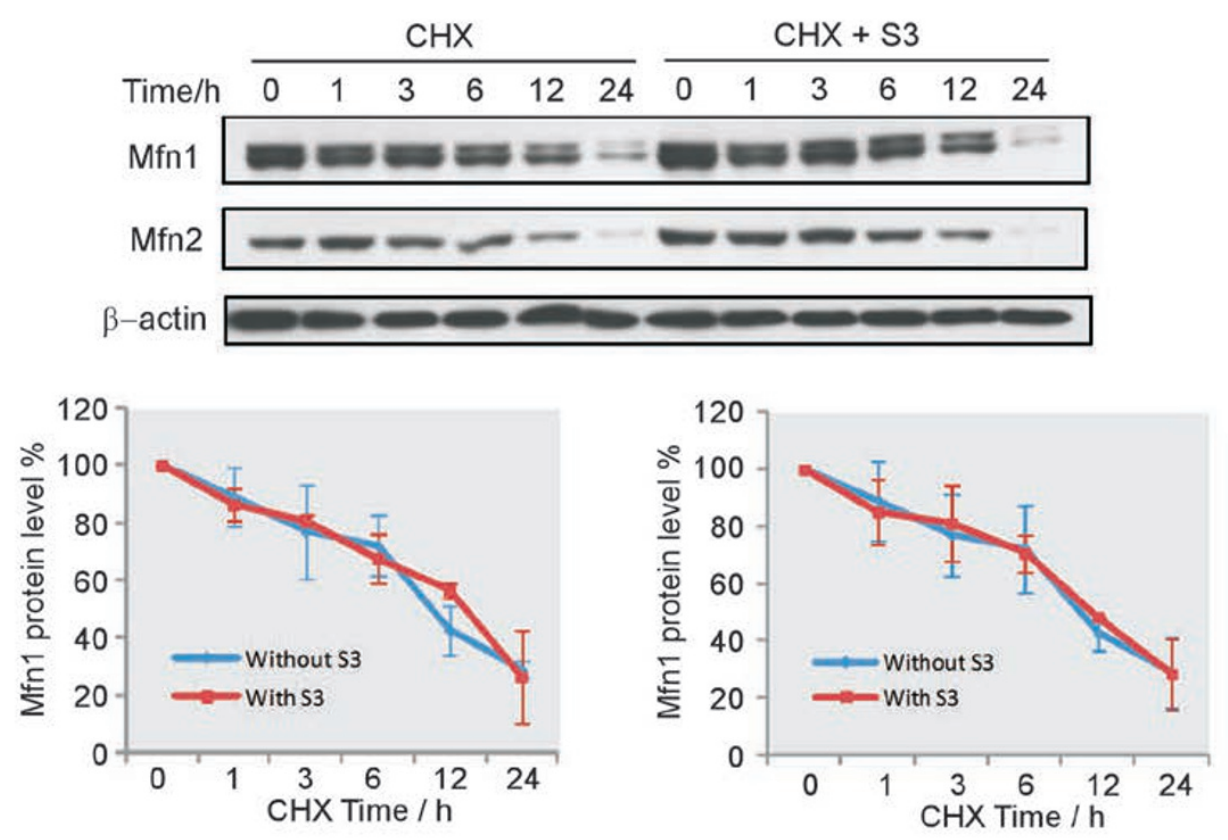

D

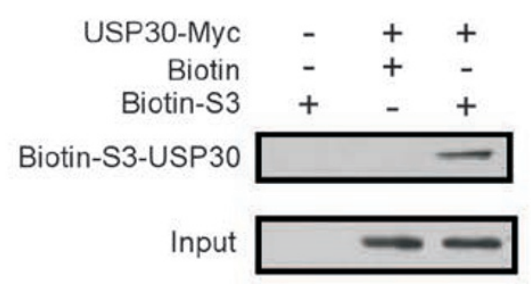

E

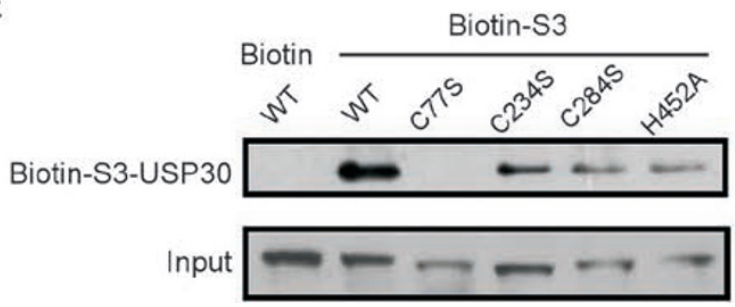

Figure $4 \mathrm{~S} 3$ targets mitochondrial deubiquitinase USP30. (A) Expression levels of Mfn1 and Mfn2 after S3 treatment. Cell lysates of WT MEF cells untreated or treated by $2 \mu \mathrm{M} \mathrm{S} 3$ for the indicated time were subjected to western blotting using antiMfn1 and anti-Mfn2 antibodies. (B) Lysates of WT MEF cells untreated or treated by $2 \mu \mathrm{M} \mathrm{S3}$ for $24 \mathrm{~h}$ were immunoprecipitated with anti-Mfn1 or anti-Mfn2 antibody. Immunoprecipitates were analyzed by western blotting for checking the ubiquitination level of Mfn1 or Mfn2. (C) CHX chase assay in S3-treated or untreated cells. Cells were treated with $\mathrm{CHX}$ or $\mathrm{CHX}$ plus 2 $\mu \mathrm{M}$ S3 for 0-24 h. The whole cell lysates were analyzed by immunoblotting with anti-Mfn1 and anti-Mfn2 antibodies. $\beta$-actin was used as loading control. The quantitative results of Mfn1 and Mfn2 protein levels were analyzed by the IMAGE J software. The $\beta$-actin-normalized Mfn1 or Mfn2 protein level at $0 \mathrm{~h}$ was defined as $100 \%$. Data shown represent mean $\pm \mathrm{SD}$ of at least two independent experiments. (D) The direct interaction of USP30 and S3. HeLa cells expressing USP30-Myc were lysed and then incubated with Biotin-S3. Biotin-S3-bound proteins were separated using streptavidin agarose resin. USP30Myc was detected by anti-Myc antibody. (E) The interactions of USP30 mutants and Biotin-S3. Indicated mutants of USP30Myc were expressed in HeLa cells. Biotin-S3 was added to the cell lysates and streptavidin agarose was used to separate Biotin-S3-bound proteins. 
A

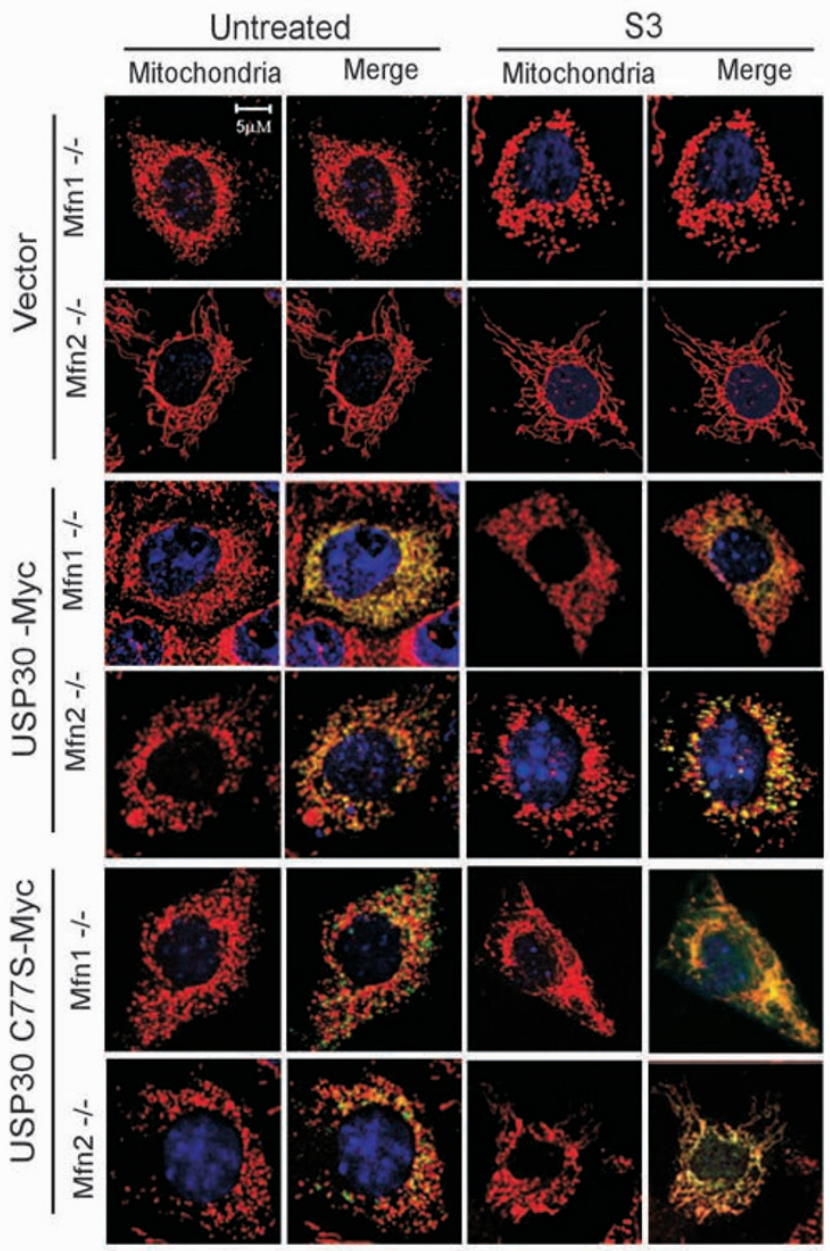

B

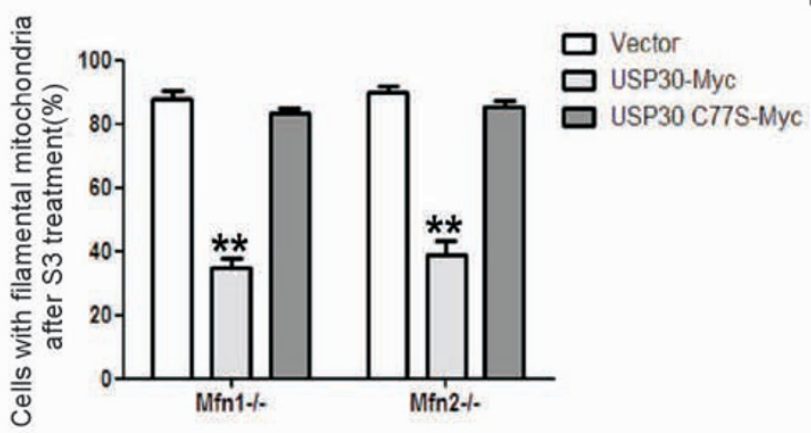

C
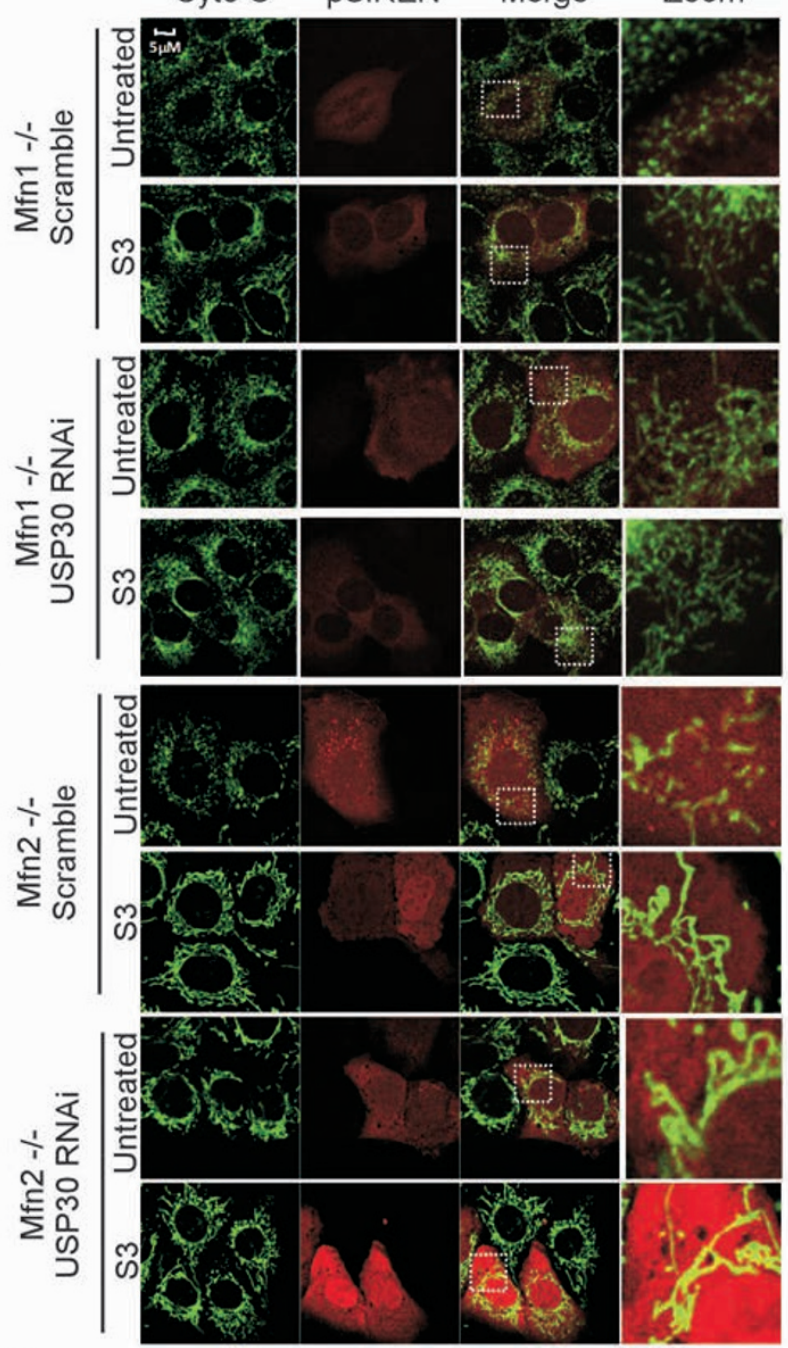

D

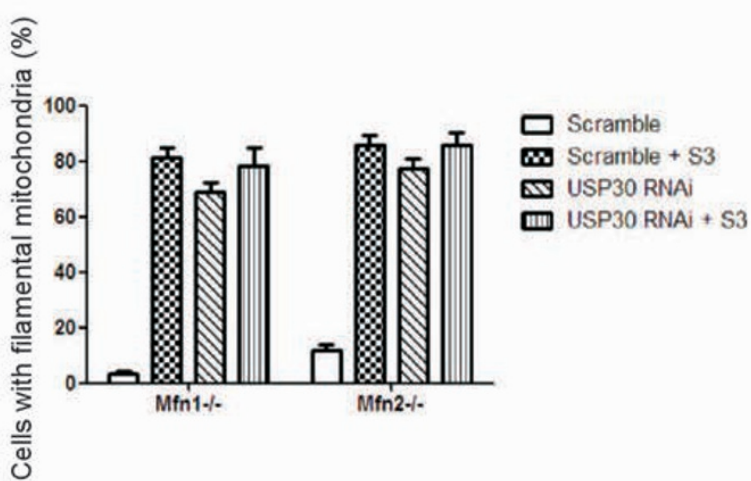

Figure 5 The effect of USP30 alteration on S3-induced mitochondrial fusion. (A) Overexpression of USP30 attenuates mitochondrial fusion induced by S3. Mfn $1^{-/}$and $\mathrm{Mfn}^{-{ }^{--}}$cells were transfected with empty vector or USP30-Myc or USP30-C77S -Myc and then left untreated or treated with $2 \mu \mathrm{M} \mathrm{S3}$ for $24 \mathrm{~h}$. Cells were stained with MitoTracker and immunostained with Myc antibody (green). (B) Statistical analysis of cells with connected and long mitochondria in A. Data shown represent mean $\pm \mathrm{SD}$ of at least three independent experiments, each with $>200$ cells analyzed per condition. ${ }^{*} P<0.001$, unpaired $t$ test, two tailed. (C) Mitochondrial morphology in USP30-RNAi cells. Mfn1 $1^{-/-}$and Mfn2 $2^{-/-}$MEF cells were transfected with pSIRENDsRed-USP30-RNAi (red) and immunostained with anti-cytochrome $c$ antibody (green) to show the morphology of mitochondria. (D) Statistical analysis of cells with connected and long mitochondria in C. Data shown represent mean \pm SD of at least three independent experiments, each with > 100 cells analyzed per condition. 
resembles the effect of S3 treatment. Approximately, $70 \%$ of $\mathrm{Mfn} 1^{-/-}$cells and $75 \%$ of $\mathrm{Mfn} 2^{-/-}$cells contain filamental mitochondria upon USP30-shRNA transfection, compared to $3 \%$ in scramble-transfected Mfn $1^{-/-}$and $11 \%$ in scramble-transfected $\mathrm{Mfn} 2^{-/-}$ cells. However, S3 treatment cannot further increase the percentage of cells with filamental mitochondria in USP30-shRNA-tranfected $\mathrm{Mfn}^{-/-}$or $\mathrm{Mfn}^{-/-}$cells, indicating that depletion of USP30 is sufficient to render cells insensitive to $\mathrm{S} 3$ treatment. Collectively, these data clearly indicate that USP30 is a target of S3, and is responsible for mediating S3-induced mitochondrial fusion in $\mathrm{Mfn}^{-/-}$or Mfn2 $2^{-/-}$cells.

We also tested whether knockdown of USP30 can enhance mitochondrial function as S3 did. We found that in both $\mathrm{Mfn}^{-/-}$and $\mathrm{Mfn}^{-/-}$cells, knockdown of USP30 recovered the mitochondrial network along with the restoration of the overall $\Delta \psi \mathrm{m}$, as indicated by the complete colocalization of Mitotracker Red and mitoGFP (Supplementary information, Figure S7B). Furthermore, the cellular ATP levels increased in both cell lines after knockdown of USP30 (Supplementary information, Figure S7C). These results indicate that USP30-knockdown cells have similar phenotypes in mitochondrial function as S3-treated cells.

\section{USP30 regulates non-degradative ubiquitination of} $M f n 1 / 2$

We next investigated whether USP30 regulates nondegradative ubiquitination of Mfn1 and Mfn2. We first examined whether USP30 had a physical interaction with Mfn1 or Mfn2. Immunoprecipitation was performed using lysates from HeLa cells overexpressing c-Myctagged USP30, and endogenous Mfn1 and Mfn2 were found to interact with USP30-Myc (Figure 6A). A pulldown assay showed that purified USP30-MBP protein can pull down Mfn1 or Mfn2 from the whole cell lysates, suggesting a direct interaction (Figure 6B). Importantly, we also found that USP30 could affect the ubiquitination levels of Mfn1 and Mfn2. When USP30 was overexpressed in HeLa cells, the ubiquitination levels of both Mfn1 and Mfn2 were obviously decreased compared to vector-transfected control cells (Figure 6C). In contrast, the ubiquitination levels of both $\mathrm{Mfn} 1$ and Mfn2 were not altered in cells expressing USP30-C77S. To further demonstrate that the regulatory function of USP30 in ubiquitination of Mfn1 and Mfn2 is specific, we performed rescue experiments. We stably knocked down USP30 by using RNAi in HeLa cells and then reintroduced the RNAi-resistant WT USP30 (Figure 6C). Indeed, ubiquitination of Mfn1 and Mfn2 significantly increased in USP30-RNAi cells and the ubiquitination of Mfn1 and Mfn2 was recovered to the levels similar to those in control cells when USP30 was reintroduced into these cells. The overall protein levels of Mfn1 and Mfn2 were not changed when USP30 expression level was altered.

To further confirm that USP30-regulated ubiquitination is non-degradative, we performed $\mathrm{CHX}$ chase assay in USP30-overexpressing and -knockdown cells. Our results showed that there was no significant difference in the degradation rate of Mfn1 and Mfn2 when USP30 was overexpressed or knocked down (Figure 6D and $6 \mathrm{E})$. These results suggest that $\mathrm{S} 3$-induced ubiquitination of Mfn1 and Mfn2 does not lead to protein degradation. Taken together, we conclude that USP30 can regulate the non-proteolytic ubiquitination of Mfn1 and Mfn2.

\section{Discussion}

In the current study, we first identified a small natural compound, 15-oxospiramilactone (S3) derived from spiramine A of Spiraea japonica, which potently induces mitochondrial fusion. In previous studies, high concentrations of S3 were found to induce apoptosis, through inhibition of the Wnt pathway [40] or upregulation of Bim [46]. However, at a low concentration, such as 2 $\mu \mathrm{M}$, S3 does not induce apoptosis, indicating a completely different mechanism for the mitochondrial fusion induced by S3. S3-induced mitochondrial fusion is dependent on the existing fusion factors on both the outer and inner mitochondrial membranes as S3 treatment fails to to induce fusion in the absence of both Mfns or OPA1. To the best of our knowledge, S3 is the first identified naturally derived small molecule from plants that activates mitochondrial fusion and facilitates restoration of the mitochondrial network. Several chemical compounds including Mdivi-1, 15d-PGJ2 and ethacrinic acid have been reported to induce mitochondrial elongation through the inhibition of mitochondrial fission mediated by Drp1 [47-51], which differs from the mechanism of S3-induced mitochondrial fusion as S3 does not inhibit Drp1 activity or phosphorylation (Supplementary information, Figure S4). However, Drp1 is not the only molecule involved in mitochondrial fission, whether S3 affects other molecular pathways in mitochondrial fission needs further investigation.

Recently, Tondera et al. [41] reported that severe cell stresses, such as mRNA translation inhibition, UV irradiation and actinomycin D treatment that downregulates protein synthesis, can trigger mitochondrial fusion in an OPA1-, Mfn1- but not Mfn2-dependent manner. This stress-induced mitochondria hyperfusion (SIMH) is dependent on the presence of stomatin-like protein 2 (SLP2). 
A

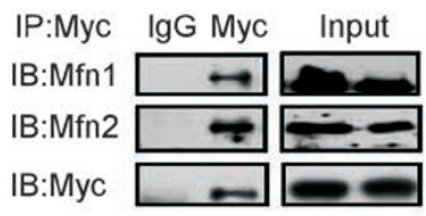

B

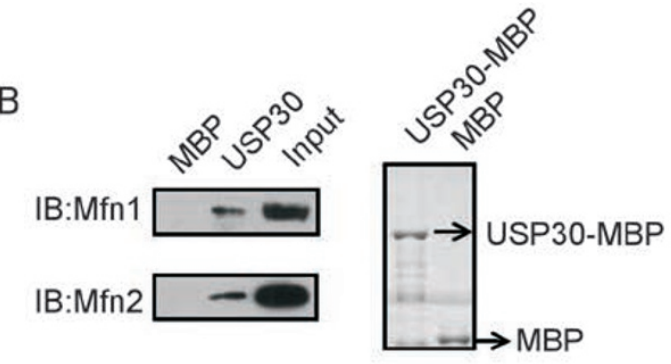

C
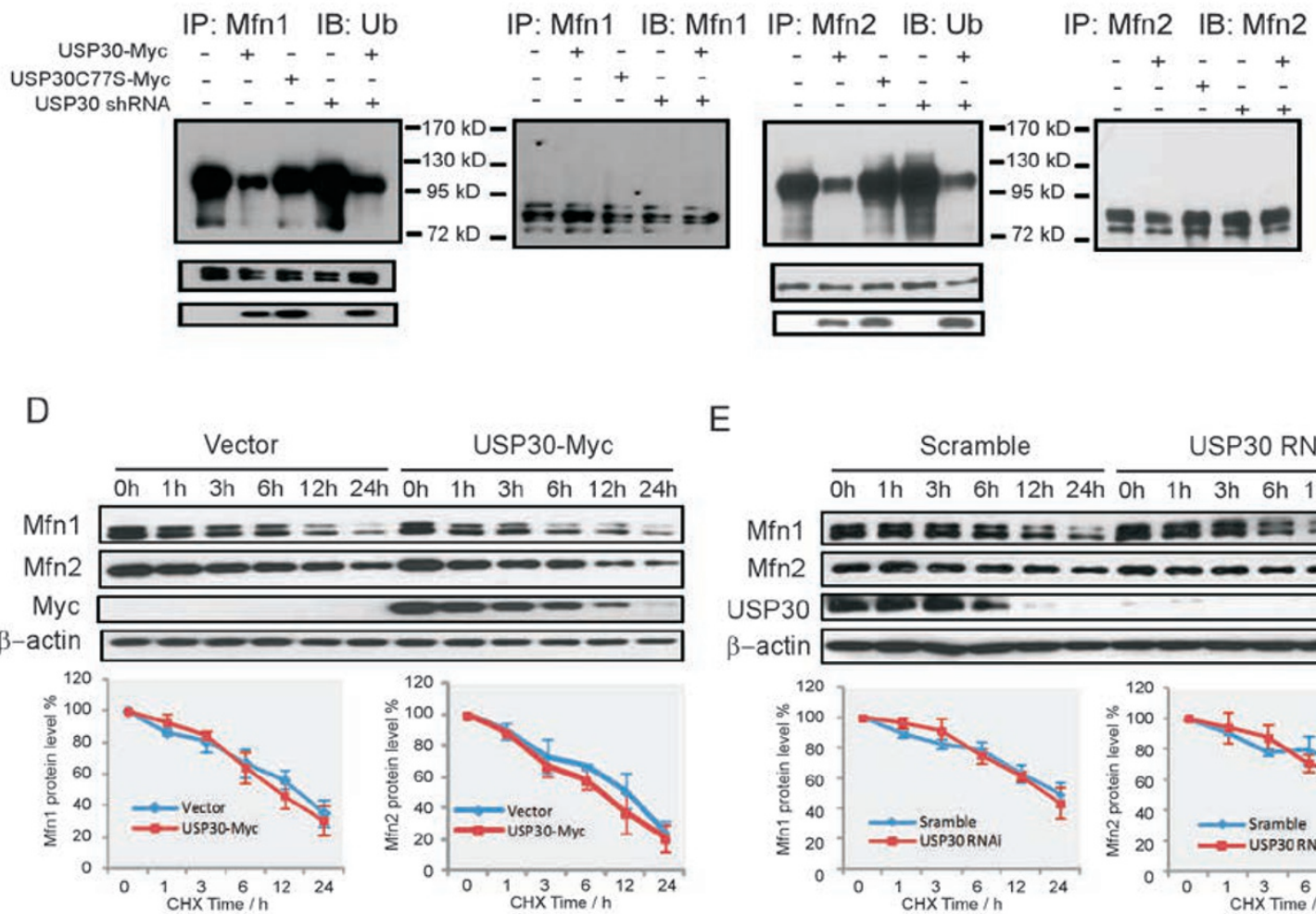

$\frac{\text { USP30-Myc }}{\text { Oh } 1 \mathrm{~h} 3 \mathrm{~h} 6 \mathrm{~h} 12 \mathrm{~h} 24 \mathrm{~h}}$

E
Scramble Oh 1h 3h 6h $12 \mathrm{~h} 24 \mathrm{~h}$ oh $1 \mathrm{~h} 3 \mathrm{~h} 6 \mathrm{~h} 12 \mathrm{~h} 24 \mathrm{~h}$
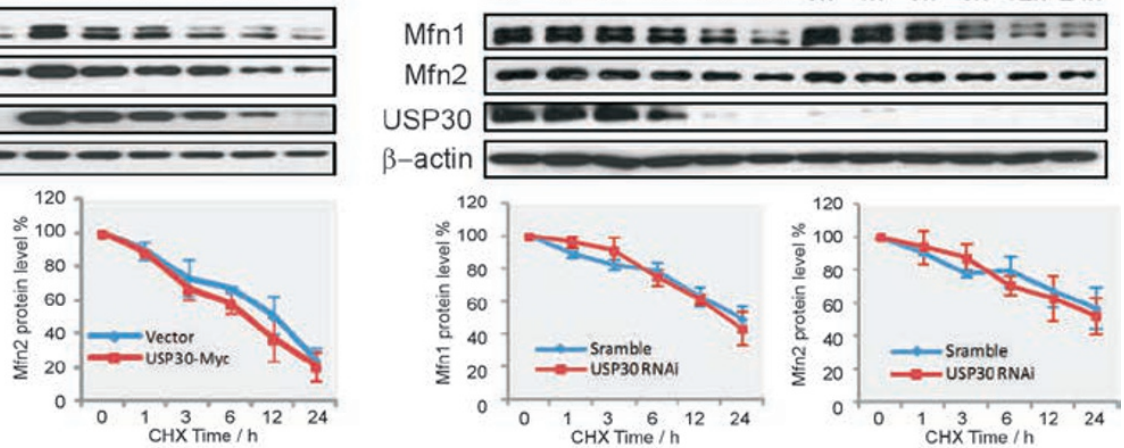

$\beta$-actin
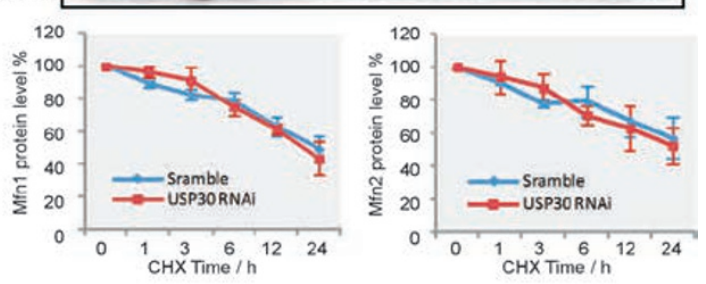

Figure 6 USP30 mediates deubiquitination of Mfn1 and Mfn2. (A) Interaction between USP30 and Mfn1/2. Lysates of HeLa cells expressing USP30-Myc were immunoprecipitated with anti-Myc antibody. Immunoprecipitates were analyzed by western blotting for Mfn1 or Mfn2. (B) Left: amylose resin with USP30-MBP protein was incubated with lysates of WT MEF cells for 3 $\mathrm{h}$ at $4{ }^{\circ} \mathrm{C}$. After washing five times, loading buffer was added and the samples were boiled for 5 min. The samples were then subjected to western blotting using anti-Mfn1 and anti-Mfn2 antibodies. Right: The same samples were subjected to SDS-PAGE analysis and stained with Coomassie Blue to confirm that USP30-MBP and MBP bound to the amylose resin. (C) Lysates of the control HeLa cells, HeLa cells expressing USP30-Myc or USP30-C77S-Myc, stable USP30-RNAi HeLa cells or USP30-RNAi cells in which USP30 were reintroduced were immunoprecipitated with anti-Mfn1 or anti-Mfn2 antibody. Immunoprecipitates were analyzed by western blotting using anti-Ubiquitin for detecting the ubiquitination of Mfn1 or Mfn2. (D, E) CHX chase assay in USP30-overexpressing cells (D) and USP30-knockdown cells (E). Cells expressing USP30-Myc (D) and stable USP30-RNAi HeLa cells or scramble-transfected cells (E) were treated with $\mathrm{CHX}$ for 0-24 h. The whole cell lysates were analyzed by immunoblotting with anti-Mfn1 and anti-Mfn2 antibodies. $\beta$-actin was used as loading control. The quantitative results of Mfn1 and Mfn2 protein levels were analyzed by IMAGE J software. The $\beta$-actin-normalized Mfn1 or Mfn2 protein level at $0 \mathrm{~h}$ was defined as $100 \%$. Data shown represent mean \pm SD of at least two independent experiments.

However, S3-induced fusion depends on either Mfn1 or Mfn2 and does not induce the degradation of proteins we examined, and knockdown of SLP2 did not affect the mitochondrial fusion induced by S3 (Supplementary information, Figure S8). These results indicate that S3induced mitochondrial fusion is different from SIMH.

Using this novel compound as a tool, we further uncovered a novel regulatory mechanism of mitochondrial 
fusion through the inhibition of a mitochondria-localized deubiquitinase USP30. We found that S3, which is a "thio-Michael addition" agent, can specifically react with the cysteine residue in the catalytic domain of USP30 to inhibit its deubiquitinase activity. UPS30 physically interacts with Mfns and decreases ubiquitination of Mfn1 and Mfn2, whereas mutation of the active cysteine (C77) in the catalytic domain to serine abolishes its deubiquitinase activity, and thus the USP30-C77S mutant is unable to affect the ubiquitination of Mfns. Our data are in agreement with a previous study showing that disruption of USP30 resulted in mitochondrial elongation in an Mfn1/2-dependent manner [45]. Furthermore, our data suggests a novel mechanism by which this deubiquitinase regulates mitochondrial fusion. Specifically, we showed that inhibition of USP30 could lead to enhanced ubiquitination of Mfn1 or Mfn2, which promotes mitochondrial fusion. It is worth noting that S3 treatment remarkably increases the ubiquitination levels of both proteins without downregulating their protein levels, indicating that this specific ubiquitination is not related to protein degradation but to functional regulation. Ubiqutinationmediated protein-protein interactions have been demonstrated to play a key role for diverse cellular functions [31]. Recently, ubiquitination has been shown to regulate intermolecular interaction during mitochondrial fusion. Anton et al. [52] found that the yeast homolog of human USP15, Ubp12, could modulate non-proteolytic ubiquitination and fusion activity of Fzol (yeast homolog of Mfns). Whether USP15 plays a similar role in mammalian cells remains unknown and merits further exploration. Nonetheless, our data indicate a previously undescribed regulatory mechanism of mitochondrial fusion, which involves USP30-regulated non-proteolytic ubiquitination of Mfns. More exploration is needed to further elucidate the comprehensive molecular mechanism of mitochondrial fusion regulated by non-degradative ubiquitination in mammalian cells.

Our results show that $\mathrm{S} 3$ can effectively trigger mitochondrial fusion to restore mitochondrial functions. It has been shown that a significant portion of individual mitochondria exhibited a loss of mtDNA and membrane potential in Mfns- or OPA1-deficient cells [10, 42, 53]. $\mathrm{S} 3$ can restore the normal distribution of mtDNA and recover the $\Delta \psi \mathrm{m}$. Thus, S3 increases the ATP level and OXPHOS capacity within the rebuilt mitochondrial network. The restoration of mitochondrial function and mtDNA abundance is important as abnormality of mitochondrial fusion is closely linked with their pathophysiologic functions; for example, mutations in Mfn 2 or OPA1 are known to cause severe neurodegenerative diseases $[14,15]$. Thus, S3 has therapeutic implications for treat- ment of these non-curable diseases related to defects in mitochondrial dynamics.

\section{Materials and Methods}

\section{Cell culture and transfection}

HeLa cells, WT, Mfn $1^{-/}$, Mfn2 $2^{-/}$, Mfn Dko and OPA1 ${ }^{-/}$MEF cells were cultured in complete Dulbecco's minimum essential medium supplemented with $10 \%$ heat inactivated fetal calf serum, 100 $\mathrm{U} / \mathrm{ml}$ penicillin and $100 \mu \mathrm{g} / \mathrm{ml}$ streptomycin in $5 \% \mathrm{CO}_{2}$ at $37{ }^{\circ} \mathrm{C}$. Transfections were performed using Lipofectamine 2000 (Invitrogen) according to the manufacturers instructions.

\section{Chemicals and antibodies}

The following antibodies were used: anti-Mfn1 and anti-Mfn2 monoclonal antibody (Abnova); anti-cytochrome c antibody (BD Biosciences); anti-ubiquitin (P4D1) and anti-c-Myc monoclonal antibody (Santa Cruz); anti-USP30 polyclonal antibody (AVIVA); and anti-DNA monoclonal antibody (Millipore).

MitoTracker Red was purchased from Invitrogen. All other reagents were from Sigma (St. Louis, MO, USA) unless otherwise stated.

\section{Vectors}

A cDNA encoding mouse USP30 was obtained from a mouse skeletal muscle library by polymerase chain reaction amplification and then inserted into the Kpnl-Xhol sites of pCDNA4/TO/MycHis (Invitrogen) to generate a USP30-Myc plasmid. USP30-MBP was constructed by cloning the cDNA fragment encoding residues 57-517 of USP30 into the BamHI and HindIII site of pMAL-c2X (New England Biolabs), respectively. The pSilencer2.1-U6 PuroUSP30 RNAi was a kind gift from Shigehisa Hirose (Tokyo Institute of Technology, Japan). The Mito-PAGFP plasmid was a kind gift from Jean-Claude Martinou (University of Geneva, Switzerland).

\section{Quantification of mitochondrial morphology}

To visualize the mitochondrial network with MitoTracker Red staining, cells grown on coverslips were incubated in a growth medium supplemented with $50 \mathrm{nM}$ MitoTracker Red for $20 \mathrm{~min}$, washed in fresh warm medium and fixed with $4 \%$ methyl aldehyde solution for $15 \mathrm{~min}$. The coverslips were washed three times with PBS and visualized using an LSM510 meta confocal microscope (Zeiss, Germany). Cells displaying a highly interconnected, tubular mitochondrial network $(>50 \%$ of the mitochondria in the whole cell population) were counted.

\section{Immunocytochemistry and transfection}

For immunocytochemistry, MEF cells were grown on collagencoated slides and transfected with pCDNA4/TO-USP30-Myc or USP30-C77S-Myc. Cells were transfected using Lipofectamine 2000 according to the manufacturers instructions. Twenty four hour after transfection, cells were treated with $2 \mu \mathrm{M} \mathrm{S} 3$ for another $24 \mathrm{~h}$. After staining with Mitotracker Red, cells were washed with PBS, fixed with $4 \%$ (wt/vol) PFA in PBS for 15 min and permeabilized with $0.2 \%$ (vol $/ \mathrm{vol}$ ) Triton X-100 for $10 \mathrm{~min}$. After washing with PBS, cells were labeled at $4{ }^{\circ} \mathrm{C}$ overnight with a c-Myc antibody. The next day, cells were washed with PBS and labeled with a combination of FITC-conjugated anti-mouse secondary antibod- 
ies (Dianova, Germany). Cell nuclei were stained with the fluorescent chromatin dye Hoechst 33342 (Molecular Probes, USA).

\section{Electron microscopy}

S3-treated or untreated cells were fixed for $2 \mathrm{~h}$ at room temperature (RT) in a culture medium supplemented with $2.5 \%$ glutaraldehyde. After washing in $100 \mathrm{mM}$ phosphate buffer $\left(\mathrm{KH}_{2} /\right.$ $\mathrm{Na}_{2} \mathrm{HPO}_{4}$, pH 7.4), cells were post-fixed for $60 \mathrm{~min}$ at RT in $1 \%$ osmium tetroxide $\left(\mathrm{OSO}_{4}\right)$. After washing in phosphate buffer, cells were dehydrated in 50, 70, 80, 90 and $100 \%$ ethanol (for 8 min for each procedure). The samples were then infiltrated sequentially in 1:1 (vol/vol) ethanol:Spurr resin (Polyscience), 1:3 ethanol:Spurr resin for $30 \mathrm{~min}$ for each procedure, $100 \%$ Spurr resin for $3 \mathrm{~h}$ and finally $100 \%$ Spurr resin for $24 \mathrm{~h}$ at $60{ }^{\circ} \mathrm{C}$ for polymerization. Ultra-thin sections were isolated on nickel grids and stained for 10 min in $2 \%$ uranyl acetate and for 5 min in Reynold's lead citrate, and examined at $60 \mathrm{kV}$ using a Philips M400 transmission electron microscope.

\section{Immunoprecipitation}

Cells were lysed in 1\% (v/v) NP40 in PBS supplemented with protease inhibitors, then c-Myc, Mfn-1 or Mfn-2 antibodies were added and the mixture rotated at $4{ }^{\circ} \mathrm{C}$ for $4 \mathrm{~h}$. A total of $20 \mu \mathrm{l}$ Protein $\mathrm{G}$ sepaharose (Santa Cruz) was then added and the mixture rotated at $4{ }^{\circ} \mathrm{C}$ for a further $4 \mathrm{~h}$. The antibody-bound beads were washed three times with PBS and then resuspended in $2 \times$ loading buffer containing $0.1 \mathrm{M}$ DTT. After boiling, the supernatant was then separated by SDS-PAGE and transferred to a nitrocellulose filter membrane for western blotting.

\section{Measurement of ATP levels}

ATP levels were measured using the ATP Determination Kit (Beyotime Institute of Biotechonology) according to the manufacturers protocol.

\section{Analysis of protein expression}

Briefly, cells were washed and lysed in a lysis buffer containing $150 \mathrm{mMNaCl}, 25 \mathrm{mM}$ HEPES pH 7.4, 1\% NP40, 0.25\% sodium deoxycholate, $1 \mathrm{mM}$ EGTA, $1 \mathrm{mM}$ DTT, with protease inhibitors. Proteins from total cell lysates were resolved on SDS-PAGE and transferred to a nitrocellulose membrane. The membranes were blocked with $5 \%$ non-fat dry milk and $0.1 \%$ Tween-20 for $2 \mathrm{~h}$ at RT and incubated with the indicated antibodies at $4{ }^{\circ} \mathrm{C}$ overnight. Immune complexes were detected with a HRP-conjugated secondary antibody and visualized by ECL (Pierce).

\section{Biotin-S3 pull-down assay}

Cells were washed and lysed in a lysis buffer on ice for $30 \mathrm{~min}$. After centrifugation, the supernatant was incubated with $0.5 \mathrm{mM}$ Biotin-S3 or Biotin at RT for $30 \mathrm{~min}$, and then precipitated with ice-cold acetone to remove excess Biotin-S3. The air-dried pellet was dissolved in a $150 \mu \mathrm{l}$ denaturing buffer ( $25 \mathrm{mM}$ HEPES $\mathrm{pH}$ 7.7, 2 mM EDTA, $2.5 \%$ SDS). Two volumes of binding buffer ( $25 \mathrm{mM}$ HEPES pH 7.7, 2 mM EDTA, 1\% Triton X-100, $150 \mathrm{mM} \mathrm{NaCl}$ ) with $50 \mu 1$ streptavidin-agarose beads (Pierce) was added and incubated for $3 \mathrm{~h}$ at RT. The resin was washed five times with a washing buffer (25 mM HEPES pH 7.7, 2 mM EDTA, 0.5\% Triton $\mathrm{X}-100,600 \mathrm{mM} \mathrm{NaCl}$ ), boiled in SDS-PAGE loading buffer for 5 min, and subjected to western blot analysis.

\section{Protein purification}

The BL21(DE3) bacteria harboring expression plasmid of the $\mathrm{N}$-terminal MBP-tagged USP30 was cultured in LB containing 20 $\mu \mathrm{g} / \mathrm{ml}$ ampicillin. Isopropyl thiogalactoside $(0.3 \mathrm{mM})$ was added and the cells were cultured for another $12 \mathrm{~h}$ at $18{ }^{\circ} \mathrm{C}$. The bacterial cells were recovered and subjected to sonification in a lysis buffer $(0.1 \%$ Triton X-100 in PBS and protease inhibitors). The lysate was centrifuged at $10000 \mathrm{~g}$ for $30 \mathrm{~min}$ and the supernatant was incubated with Amylose Resin (New England Biolabs) for $2 \mathrm{~h}$ at RT. The resin was washed with PBS until excess proteins were completely removed. The USP30-MBP was eluted with $10 \mathrm{mM}$ maltose in PBS. In total, $200 \mu \mathrm{g}$ of USP30-MBP was cleaved by 5 $\mu 1$ Factor Xa at $4{ }^{\circ} \mathrm{C}$ for $24 \mathrm{~h}$ and the cleaved USP30 were used to detect deubiquitinase activity in vitro.

\section{Statistical analysis}

In quantitative analyses using cultured cells represented as histograms, values were obtained from three independent experiments and expressed as mean \pm SD. Statistical analysis was performed using the Student's $t$ test, with $P$-values $<0.05$ considered significant. ${ }^{*} P<0.05$ and $* * P<0.01$ versus the corresponding controls are indicated in the figures. All statistical data were calculated with the GraphPad Prism software.

\section{Acknowledgments}

We thank David C Chan (California Institute of Technology, USA) for generously providing us with the $\mathrm{Mfn}^{-/-}, \mathrm{Mfn}^{-/-}$and $\mathrm{OPA}^{-/-}$cells and Mfn Dko cells. We thank Mark Bartlam (Nankai University, China) and Aiming Zhou (Cleveland State University Cleveland, USA) for their help on the writing. Research in Chen's laboratory was supported by the Natural Science Foundation of China (NSFC; 91213304 and 81130045), the Ministry of Sciences and Technology (2011CB910903 and 2010CB912204) and the NSFC-joint foundation of Yunnan Province (U1032603).

\section{References}

1 Chan DC. Mitochondria: dynamic organelles in disease, aging, and development. Cell 2006; 125:1241-1252.

2 Hoppins S, Lackner L, Nunnari J. The machines that divide and fuse mitochondria. Ann Rev Biochem 2007; 76:751-780.

3 Santel A, Fuller MT. Control of mitochondrial morphology by a human mitofusin. J Cell Sci 2001; 114:867-874.

4 Koshiba T, Detmer SA, Kaiser JT, Chen H, McCaffery JM, Chan DC. Structural basis of mitochondrial tethering by mitofusin complexes. Science 2004; 305:858-862.

5 Ishihara N, Eura Y, Mihara K. Mitofusin 1 and 2 play distinct roles in mitochondrial fusion reactions via GTPase activity. $J$ Cell Sci 2004; 117:6535-6546.

6 Hoppins S, Edlich F, Cleland MM, et al. The soluble form of Bax regulates mitochondrial fusion via MFN2 homotypic complexes. Mol Cell 2011; 41:150-160.

7 Detmer SA, Chan DC. Complementation between mouse Mfn1 and Mfn2 protects mitochondrial fusion defects caused by CMT2A disease mutations. J Cell Biol 2007; 176:405-414.

8 Wong ED, Wagner JA, Gorsich SW, McCaffery JM, Shaw JM, Nunnari J. The dynamin-related GTPase, Mgm1p, is an intermembrane space protein required for maintenance of fu- 
sion competent mitochondria. J Cell Biol 2000; 151:341-352.

9 Cipolat S, Martins de Brito O, Dal Zilio B, Scorrano L. OPA1 requires mitofusin 1 to promote mitochondrial fusion. Proc Natl Acad Sci USA 2004; 101:15927-15932.

10 Chen H, Chomyn A, Chan DC. Disruption of fusion results in mitochondrial heterogeneity and dysfunction. $\mathrm{J}$ Biol Chem 2005; 280:26185-26192.

11 Detmer SA, Chan DC. Functions and dysfunctions of mitochondrial dynamics. Nat Rev Mol Cell Biol 2007; 8:870-879.

12 Chen H, Detmer, SA, Ewald AJ, Griffin EE, Fraser SE, Chan DC. Mitofusins Mfn1 and Mfn2 coordinately regulate mitochondrial fusion and are essential for embryonic development. J Cell Biol 2003; 160:189-200.

13 Cipolat S, Rudka T, Hartmann D, et al. Mitochondrial rhomboid PARL regulates cytochrome c release during apoptosis via OPA1-dependent cristae remodeling. Cell 2006; 126:163175.

14 Zuchner S, Mersiyanova IV, Muglia M, et al. Mutations in the mitochondrial GTPase mitofusin 2 cause Charcot-MarieTooth neuropathy type 2A. Nat Genet 2004; 36:449-451.

15 Alexander C, Votruba M, Pesch UE, et al. OPA1, encoding a dynamin-related GTPase, is mutated in autosomal dominant optic atrophy linked to chromosome 3q28. Nat Genet 2000; 26:211-215.

16 Smirnova E, Shurland DL, Ryazantsev SN, van der Bliek AM. A human dynamin-related protein controls the distribution of mitochondria. J Cell Biol 1998; 143:351-358.

17 Ingerman E, Edward M. Perkins, Michael Marino, et al. Dnm1 forms spirals that are structurally tailored to fit mitochondria. J Cell Biol 2005; 170:1021-1027.

18 Otera $\mathrm{H}$, Wang C, Cleland MM, et al. Mff is an essential factor for mitochondrial recruitment of Drp1 during mitochondrial fission in mammalian cells. J Cell Biol 2010; 191:11411158.

19 Palmer CS, Osellame LD, Laine D, Koutsopoulos OS, Frazier AE, Ryan MT. MiD49 and MiD51, new components of the mitochondrial fission machinery. EMBO Rep 2011; 12:565573

20 Ishihara N, Nomura M, Jofuku A, et al. Mitochondrial fission factor Drp1 is essential for embryonic development and synapse formation in mice. Nat Cell Biol 2009; 11:958-966.

21 Eura Y, Ishihara N, Oka T, Mihara K. Identification of a novel protein that regulates mitochondrial fusion by modulating mitofusin (Mfn) protein function. J Cell Sci 2006; 119:49134925.

22 Fisk HA, Yaffe MP. A role for ubiquitination in mitochondrial inheritance in Saccharomyces cerevisiae. J Cell Biol 1999; 145:1199-1208.

23 Karbowski M, Neutzner A, Youle RJ. The mitochondrial E3 ubiquitin ligase MARCH5 is required for Drp1 dependent mitochondrial division. J Cell Biol 2007; 178:71-84.

24 Wang H, Song P, Du L, et al. Parkin ubiquitinates Drp1 for proteasome-dependent degradation: implication of dysregulated mitochondrial dynamics in Parkinson disease. J Biol Chem 2011; 286:11649-11658.

25 Gegg ME, Cooper JM, Chau KY, Rojo M, Schapira AH, Taanman JW. Mitofusin 1 and mitofusin 2 are ubiquitinated in a PINK1/parkin-dependent manner upon induction of mitophagy. Hum Mol Genet 2010; 19:4861-4870.
26 Tanaka A, Cleland MM, Xu S, et al. Proteasome and p97 mediate mitophagy and degradation of mitofusins induced by Parkin. J Cell Biol 2010; 191:1367-1380.

27 Cohen MM, Amiott EA, Day AR, et al. Sequential requirements for the GTPase domain of the mitofusin Fzol and the ubiquitin ligase $\mathrm{SCF}^{\mathrm{Mdm} 30}$ in mitochondrial outer membrane fusion. J Cell Sci 2011; 124:1403-1410.

28 Nakamura N KY, Tokuda M, Honda S, Hirose S. MARCH$\mathrm{V}$ is a novel mitofusin 2- and Drp1-binding protein able to change mitochondrial morphology. EMBO Rep 2006; 7:10191022

29 Escobar-Henriques M WB, Langer T. Regulation of mitochondrial fusion by the F-box protein Mdm30 involves proteasome-independent turnover of Fzo1. J Cell Biol 2006; 173:645-650.

30 Fritz S WN, Westermann B. Mdm30 is an F-box protein required for maintenance of fusion-competent mitochondria in yeast. Mol Biol Cell 2003;14:2303-2313.

31 Welchman RL, Gordon C, Mayer RJ. Ubiquitin and ubiquitinlike proteins as multifunctional signals. Nat Rev Mol Cell Biol 2005; 6:599-609.

32 Woelk T, Oldrini B, Maspero E, et al. Molecular mechanisms of coupled monoubiquitination. Nat Cell Biol 2006; 8:12461254.

33 Komander D, Clague MJ, Urbe S. Breaking the chains: structure and function of the deubiquitinases. Nat Rev Mol Cell Biol 2009; 10:550-563.

34 Nijman SM, Luna-Vargas MP, Velds A, et al. A genomic and functional inventory of deubiquitinating enzymes. Cell 2005; 123:773-786.

35 Reyes-Turcu FE, Ventii KH, Wilkinson KD. Regulation and cellular roles of ubiquitin-specific deubiquitinating enzymes. Annu Rev Biochem 2009; 78:363-397.

36 Amerik AY, Hochstrasser M. Mechanism and function of deubiquitinating enzymes. Biochim Biophys Acta 2004; 1695:189-207.

37 Sacco JJ, Coulson JM, Clague MJ, Urbe S. Emerging roles of deubiquitinases in cancer-associated pathways. IUBMB Life 2010; 62:140-157.

38 Kapuria V PL, Fang D, Bornmann WG, Talpaz M, Donato NJ. Deubiquitinase inhibition by small-molecule WP1130 triggers aggresome formation and tumor cell apoptosis. Cancer Res 2010; 70:9265-9276.

39 Sun H, Kapuria V, Peterson LF, et al. Bcr-Abl ubiquitination and Usp9x inhibition block kinase signaling and promote CML cell apoptosis. Blood 2011; 117:3151-3162.

40 Wang W, Liu H, Wang S, Hao X, Li L. A diterpenoid derivative 15-oxospiramilactone inhibits Wnt/beta-catenin signaling and colon cancer cell tumorigenesis. Cell Res 2011; 21:730740 .

41 Tondera D, Grandemange S, Jourdain A, et al. SLP-2 is required for stress-induced mitochondrial hyperfusion. EMBO J 2009; 28:1589-1600.

42 Chen H, McCaffery JM, Chan DC. Mitochondrial fusion protects against neurodegeneration in the cerebellum. Cell 2007; 130:548-562.

43 Chen H, Vermulst M, Wang YE, et al. Mitochondrial fusion is required for mtDNA stability in skeletal muscle and tolerance of mtDNA mutations. Cell 2010; 141:280-289. 
44 Mariusz Karbowski, Damien Arnoult, Hsiuchen Chen, David C Chan, Carolyn L Smith, Youle RJ. Quantitation of mitochondrial dynamics by photolabeling of individual organelles shows that mitochondrial fusion is blocked during the Bax activation phase of apoptosis. J Cell Biol 2004; 164:493-499.

45 Nakamura N, Hirose S. Regulation of mitochondrial morphology by USP30, a deubiquitinating enzyme present in the mitochondrial outer Membrane. Mol Biol Cell 2008; 19:19031911.

46 Zhao L, He F, Liu H, et al. Natural diterpenoid compound elevates expression of bim protein, which interacts with antiapoptotic protein Bcl-2, converting it to proapoptotic Bax-like molecule. J Biol Chem 2012; 287:1054-1065.

47 Nandita Mishra RK, Prajjal K Singha, Manjeri A Venkatachalam, Donald G McEwen, Saikumar P. Inhibition of mitochondrial division through covalent modification of Drp1 by 15DG. Biochem Biophys Res Commun 2010; 395:17-24.

48 Ann Cassidy-Stone, Jerry E Chipuk, Elena Ingerman, et al. Chemical inhibition of the mitochondrial division dynamin reveals its role in Bax/Bak-dependent mitochondrial outer membrane permeabilization. Dev Cell 2008; 14:193-204.
49 Rekha Kar NM, Prajjal K Singha, Manjeri A Venkatachalam, Pothana Saikumar. Mitochondrial remodeling following fission inhibition by $15 \mathrm{~d}-\mathrm{PGJ} 2$ involves molecular changes in mitochondrial fusion protein OPA1. Biochem Biophys Res Commun 2010; 399:548-554.

50 Bowes T GR. Novel mitochondrial extensions provide evidence for a link between microtubule-directed movement and mitochondrial fission. Biochem Biophys Res Commun 2008; 376:40-45.

51 Soltys BJ, RS G. Changes in mitochondrial shape and distribution induced by ethacrynic acid and the transient formation of a mitochondrial reticulum. J Cell Physiol 1994; 159:281294.

52 Anton F, Dittmar G, Langer T, Escobar-Henriques M. Two deubiquitylases act on mitofusin and regulate mitochondrial fusion along independent pathways. Mol Cell 2013; 49:487498.

53 Chen H, Detmer SA, Ewald AJ, Griffin EE, Fraser SE, Chan DC. Mitofusins Mfn1 and Mfn2 coordinately regulate mitochondrial fusion and are essential for embryonic development. J Cell Biol 2003; 160:189-200.

(Supplementary information is linked to the online version of the paper on the Cell Research website.) 